\title{
The role of vitamin A and vitamin D in modulation of the immune response with a focus on innate lymphoid cells
}

\author{
TANJA DŽOPALIĆ ${ }^{\prime}$, BILJANA BOŽIĆ-NEDELJKOVIĆ ${ }^{2}$ VLADIMIR JURIŠIĆ \\ ${ }^{1}$ Department of Immunology, University of Niš, Medical Faculty, Niš, Serbia \\ ${ }^{2}$ Institute for Physiology and Biochemistry "Ivan Djaja" Belgrade, Faculty of Biology, University of Belgrade, Serbia \\ ${ }^{3}$ Faculty of Medical Sciences, University of Kragujevac, Kragujevac, Serbia
}

\begin{abstract}
The immune system with its numerous and complex interactions helps to protect the host from pathogenic microorganisms, and enables cleaning of damaged tissues. It is also associated with constant "monitoring" of the appearance of malignant cells and their elimination that can occur in the human body. Such a role depends on many factors including adequate intake of nutrients, including vitamins. The effect of vitamin supplementation on the modulation of the immune response has always been the focus of numerous studies. Vitamins $A$ and D have been shown to have the greatest immune-modulatory effect. In this review, we discuss and consider the possible roles of vitamins $A$ and $D$ on the immune response through innate and adaptive immune cells, with special focus on the cell population recently characterized as innate lymphoid cells. Recent literature data indicate that vitamin A and its metabolites modulate the balance between Th1 and Th2 immunity. In addition, vitamin D expresses protective effects on the innate immune system and inhibitory effects on adaptive immunity.
\end{abstract}

Key words: vitamin A, vitamin D, immune response, innate lymphoid cells.

(Cent Eur J Immunol 2021; 46 (2): 264-269)

\section{Introduction}

The main role of the immune system is reflected through the protection of the host from pathogenic microorganisms, cleaning of damaged tissues and permanent monitoring of malignant cells that can occur daily in the human body. Studies have shown that there is a strong heterogeneity of the immune response between individuals, primarily due to genetic and environmental factors, lifestyle, diet and interactions between these factors [1]. We cannot influence some factors, such as genetics, but we can change the rest. Diet is a variable factor that affects the function of the immune system and has been studied for several decades. This study of the effects of diet on immunity has grown into a special research branch known as nutritional immunology. The immune system depends on adequate nutrition to perform its function normally. Years of research have shown that nutritional status is closely related to host immunity and resistance to infections [2-5]. Although it is well known that nutritional deficiencies need to be diminished for the immune system to function adequately, recently emerged evidence suggest that increased intake of some nutrients, including vitamins, may help optimize the immune response and resistance to infection
[6-8]. The impact of vitamin supplementation on the modulation of the immune response has always been in the focus of numerous studies [9-12]. We focused this review on vitamins $\mathrm{A}$ and $\mathrm{D}$, since they were shown to have the greatest immunomodulatory impact. The latest literature data describing the way vitamins A and D influence the immune response through innate and adaptive immune cells, including recently described innate lymphoid cells, are summarized.

\section{Immunomodulatory potential of vitamin A}

Vitamin A belongs to the group of liposoluble vitamins. It exists in the form of retinol, retinal and retinoic acid (RA), among which RA shows the highest biological activity. These metabolites are essential in embryonic development, vision maintenance, growth, integrity of epithelial and mucosal tissue, and cellular differentiation [13]. Since humans cannot synthesize vitamin A de novo, dietary uptake is crucial. Retinol is derived from animal sources such as meat, dairy products, and fish, whereas provitamin A is derived from colorful fruits and vegetables. After absorption, both ingested forms of vitamin A

Correspondence: Vladimir Jurišić, Faculty of Medical Sciences, University of Kragujevac, Kragujevac, Serbia, phone: +38134306800, e-mail: jurisicvladimir@gmail.com

Submitted: 10.10.2020; Accepted: 3.01.2021 
are converted to retinal and RA, upon which biologic functions are expressed [14].

Retinoic acid is a ligand of the nuclear receptor protein for retinoic acid (RAR protein). The RAR family has 3 main members, $\alpha$ (isoforms $\alpha 1-2), \beta(\beta 1-4)$ and $\gamma$, with additional subtypes. Nuclear RAR acts as a ligand-activated transcription factor. RA exists in two significant derivatives: 9-cis-RA and all-trans-RA (ATRA) [15]. ATRA is the endogenous ligand RAR with the highest affinity.

Vitamin A metabolites through their effects on dendritic cells (DCs) can indirectly define the final outcome of the immune response $[10,11]$. Dendritic cells are antigen-presenting cells with key roles in the initiation and regulation of innate and adaptive immune responses [16]. Immature DCs upon antigen recognition by numerous pattern-recognition receptors (PRRs) become mature and migrate to secondary lymph organs where they interact with $\mathrm{T}$ cells and initiate the immune response [17]. Their role is crucial in differentiation of nadve $\mathrm{CD} 4^{+} \mathrm{T}$ cells into effector subsets, Th1, Th2, Th17 or regulatory T (reg) cells. Th1 and Th17 immune responses are mostly involved in the immunity against intracellular pathogens, with the promotion of autoimmunity and inflammation. Th2 immunity is activated in the presence of parasites and extracellular bacteria [18].

It has been shown that ATRA can act cell-intrinsically in the differentiation of DC precursors [19]. It is believed that the effect of ATRA on DCs is reflected through the promotion of the anti-inflammatory phenotype characteristic of intestinal DCs [20]. On the other hand, in the presence of interleukin (IL)-15, ATRA can stimulate the production of IL-12 and IL-23 by DCs, suggesting promotion of the Th1 and Th17 immune response [21]. Kim et al. demonstrated that DCs express enzymes in the vitamin A metabolic pathway, including the aldehyde dehydrogenase 1 family, member a2 (ALDH1A2) and short-chain dehydrogenase/reductase family, member 9 (DHRS9), enzymes capable of the two-step conversion of retinol into ATRA, which is subsequently released from the cell [11]. Such ATRA production can transactivate infected macrophages to elicit antimicrobial activity.

Balance between Th1 and Th2 immunity can also be modulated by vitamin A metabolites. Namely, by inducing IL-4, IL-5, and GATA-3 (the main regulatory factor for the Th2 lineage) RA promotes Th2 cell differentiation [22]. RA also favors the Th2 immune response by up-regulating the expression of the co-stimulatory molecule CD86 and the natural killer T (NKT) cell population, correlated with increased secretion of IL-4 [23]. On the other hand, inhibition of $T$-bet, a Th1 cell transcription factor, by RA contributes to down-regulation of the Th1 immune response [24].

Vitamin A can regulate the adaptive immune response through differentiation of Foxp $3^{+}$regulatory T cells as well [25]. Regulatory $\mathrm{T}$ lymphocytes (Treg) are a subpopulation of T lymphocytes that maintain immune tolerance and regulate the autoimmune response and Foxp 3 is a transcrip- tional factor essential for the differentiation and effector function of Tregs. The cytokine transforming growth factor $\beta$ (TGF- $\beta$ ) converts nadve T lymphocytes into Tregs that prevent autoimmunity. However, TGF- $\beta$ promotes the differentiation of nadve T lymphocytes into proinflammatory, IL-17-producing Th17 lymphocytes, which promote autoimmunity and inflammation. ATRA, as a key regulator of the TGF- $\beta$-dependent immune response, is able to inhibit IL-6-induced induction of proinflammatory Th17 cells and to promote Treg differentiation [26]. Depending on the concentration, RA has an opposite effect on the differentiation of Th1 and Th17 cells [27]. Namely, Rampal et al. demonstrated that high doses of RA can impair the differentiation of human Th17 and Th1 cells in vitro [28]. On the other hand, there are reports suggesting that RA was beneficial to Th1 and Th17 cell differentiation at low doses [29].

Innate lymphoid cells (ILCs) are recently described cells with an emerging role in the immune response. These cells mirror the phenotypes and cytokine profile of $\mathrm{CD}^{+}$ $\mathrm{T}$ cell subsets, and they are divided into three main subsets, ILC1, ILC2, and ILC3 mostly based on their potential to produced different cytokines [30]. It is thought that they correspond to Th1, Th2, and Th17 subsets, and therefore are involved in the immunity against intracellular bacteria, parasites, and extracellular microbes [31]. ILCs most likely regulate adaptive immunity through promotion of $\mathrm{T}$ cell activation via regulation of DCs [32]. Recent studies have shown that vitamin A can have an impact on ILC functions [33-35]. Namely, it has been shown that intestinal ILC2s expand during vitamin A deficiency and intestinal ILC3s were found to be highly abundant when vitamin A is present. Since vitamin A deficiency increases sensitivity to bacterial infection, it was proposed that balance of ILC2s vs. ILC3s is a regulatory mechanism to maintain intestinal barrier function in a vitamin A-dependent manner [35]. Similar results were obtained by another research group which demonstrated a direct effect of RA on intestinal ILC3s in transgenic mice, indicating its crucial role in the development of intestinal ILC3s and postnatally formed lymphoid tissue within the intestine [33]. In addition, ILC2s were shown to down-regulate the expression of IL-7R $\alpha$ in the presence of vitamin A, thereby providing a benefit for ILC3s in the competition of growth factors. The same study proposed vitamin A as a potential factor for conversion of intestinal ILC2s into IL-10, producing ILCreg, which contribute to establishment of intestinal tolerance [34].

Poor vitamin A status has been associated with increased occurrence and severity of infectious disease. Due to its described immunomodulatory capacity, vitamin $A$ and its metabolites have been used in animal models to mitigate autoimmune inflammation, psoriasis, and contact dermatitis [36]. In humans, vitamin A reduces morbidity and mortality of measles, infantile diarrhea, malaria and 
hand, foot and mouth disease [37-39]. Vitamin A has both promoting and regulatory roles in the innate and adaptive immunity, so it can provide enhanced defense against multiple diseases. A previous study demonstrated that 13-cis isomer RA has a dose- and time-dependent antiproliferative effect on the HL-60 promyelocytic leukemia (PML) cell line, which may facilitate the development of new pharmacological strategies in the treatment of PML [40]. Currently, there are some ongoing studies on therapeutic effects of vitamin A on preventing and treating various infectious diseases and cancer. Further studies will probably give insights into vitamin A application as therapeutics.

\section{Immunomodulatory potential of vitamin D}

Vitamin D is a pro-hormone, essential for many cellular physiological processes in the body contributing to good health. Since not so many nutrients are rich in vitamin D (fish, egg yolks, milk) it must be imported in another way. The most significant form of vitamin D is vitamin $D_{3}$, synthesized in the skin from 7-dehydrocholesterol depending on ultraviolet $\mathrm{B}$ radiation from sunlight [41]. Upon liver conversion to 25-dihydroxyvitamin $\mathrm{D}_{3}(25(\mathrm{OH})$ $\mathrm{VD}_{3}$ ), the main circulating form of vitamin $\mathrm{D}_{3}$, it metabolites in the kidney to $1,25(\mathrm{OH}) 2 \mathrm{VD}_{3}$, a physiologically active vitamin $\mathrm{D}_{3}$ metabolite. In some lesser amounts, vitamin $\mathrm{D}_{3}$ can be metabolized by $\mathrm{T}$ and $\mathrm{B}$ lymphocytes, macrophages and DCs [42]. Since many of the immune cells express vitamin D receptor (VDR) and produce 1- $\alpha$-hydroxylase, an enzyme necessary for vitamin $\mathrm{D}_{3}$ activation, this vitamin can exert its effects in an autocrine, paracrine and endocrine manner [1].

Vitamin $\mathrm{D}_{3}$ modulates the innate immune system by enhancing the phagocytic ability of immune cells and by reinforcing the physical barrier function of epithelial cells [43]. It has been shown that vitamin $\mathrm{D}_{3}$ stimulates monocyte proliferation and promotes chemotactic and phagocytic capacity of macrophages in a Toll-like receptor (TLR)-dependent manner. Namely, it seems that microbial activation of macrophages through TLR2 and TLR4 enhances 1 - $\alpha$-hydroxylase and VDR activity with subsequent cathelicidin and beta defensin 4 production. Such processes are important for intracellular bacteria killing [43]. Evidence from animal studies demonstrates that lacking vitamin D impairs gut innate immunity, leading to gut dysbiosis and systemic inflammation [44]. In addition, vitamin $\mathrm{D}_{3}$ improves protective effects of other cells participating in the innate immune system as first-barrier defenses, such as keratinocytes, epithelial, intestinal, lung and corneal cells, and placenta trophoblasts [45].

Recent studies have assessed the role of vitamin D in ILCs biology [46, 47]. Vitamin D is an important regulator of early cytokine production by ILC in the gut and therefore valuable in enteric infection protection. It has been demonstrated that the vitamin D/VDR signaling pathway critically regulates ILC 3 development in the gut of mice through IL-22 production, providing potent innate immunity against $C$. rodentium infection $[9,47]$. Interestingly, antagonistic effects of vitamins D and A in human ILCs have been observed [48]. Data show that vitamin $\mathrm{D}_{3}$ inhibits RA-induced production of several effector cytokines and expression of gut-homing integrin in human ILCs. Since ILC2s enhance allergic inflammation, their inhibition by vitamin $\mathrm{D}_{3}$ could therefore ameliorate allergic inflammation, and maybe prevent allergic disease.

In contrast to innate immunity, activity of the adaptive immune response is mostly inhibited in the presence of vitamin $\mathrm{D}_{3}$. In general, vitamin $\mathrm{D}_{3}$ inhibits $\mathrm{T}$ cell proliferation by decrease in IL-2 and interferon $\gamma$ (IFN- $\gamma$ ) production [49]. Such an effect is probably mediated through formation of a VDR-retinoic X receptor (RXR) complex to the vitamin $D_{3}$ response elements (VDREs) in the promoters of genes encoding IL-2 and IFN- $\gamma$ [36]. Vitamin D reduces $\mathrm{CD}^{+} \mathrm{T}$ cell polarization toward the pro-inflammatory $\mathrm{Th} 1$ and Th17 cells, opposite of favoring the regulatory Th2 and Treg cell development $[45,50]$. Studies indicate that vitamin $\mathrm{D}$ favors Th2 cell development by enhancement of IL-4 production, whereas down-regulation of IFN- $\gamma$ blocks Th1 cell activation [36]. The effect on Th17 cells is attributed to inhibition of IL-6 and IL-23 production with probable reciprocal differentiation and expansion of Foxp3 of Treg cells [51]. It is presumed that most of these effects of vitamin $\mathrm{D}$ on adaptive immunity are mediated through DCs [52]. Immunostimulatory capacity of DCs seems to be reduced in the presence of vitamin $\mathrm{D}_{3}$, as determined by down-expression of MHC II class molecules, CD40, CD80 and CD86 molecules, key markers of DCs [53, 54]. Vitamin $D_{3}$ simultaneously decreases the production of IL-12 and increases IL-10 production by DCs, suggesting its role in polarization of the immune response [55].

Figure 1 schematically describes simultaneous effects of vitamins D and A on effector functions of the immune cells.

Most studies examining vitamin D metabolism and disease prevalence show an association between low serum vitamin D concentrations and autoimmunity (Fig. 2). Also, different animal studies have provided evidence of an association between hypovitaminosis D and high risk of systemic lupus erythematosus, rheumatoid arthritis, systemic sclerosis, multiple sclerosis, inflammatory bowel diseases, type 1 diabetes and other autoimmune diseases [56]. The described immunomodulatory properties of vitamin D and its metabolites suggest that vitamin D supplementation could be of great help in the treatment of inflammatory and autoimmune diseases.

It is important to note that since both vitamin A and vitamin D act through a similar type of receptor, it can cause a problem in their supplementation. Namely, the nuclear VDR heterodimerizes with nuclear receptors of the RXR family and binds to VDREs, or forms RXR-RXR homodi- 


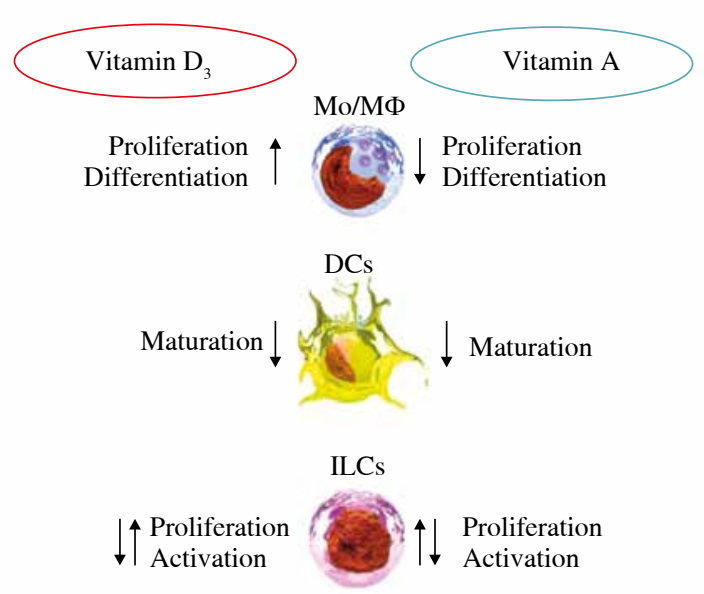

Fig. 1. Simultaneous effects of vitamins D and A on effector functions of the immune cells (influence on ILC subtypes still not definitely dear)

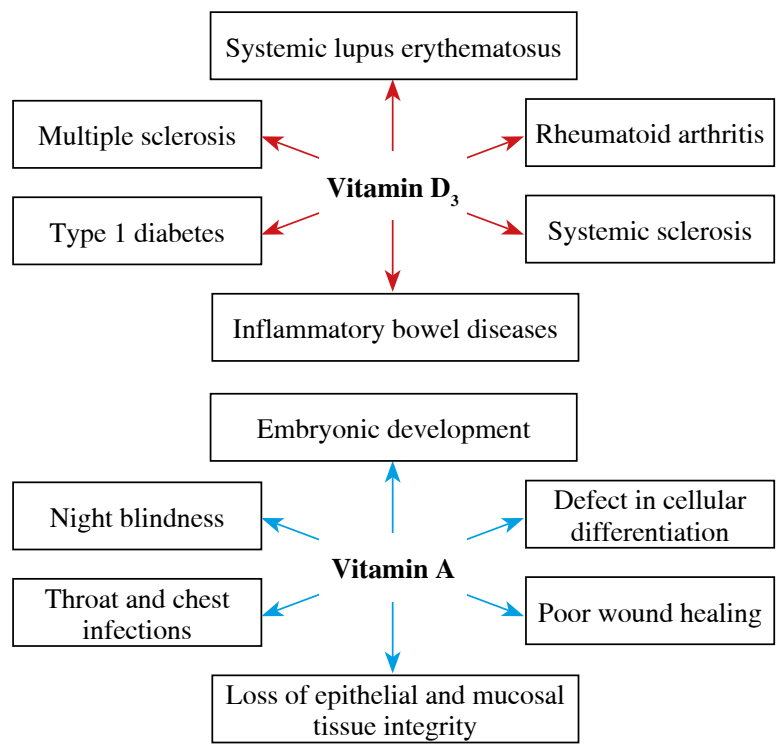

Fig. 2. The most common diseases and disorders related to vitamins $\mathrm{D}$ and A hypovitaminosis

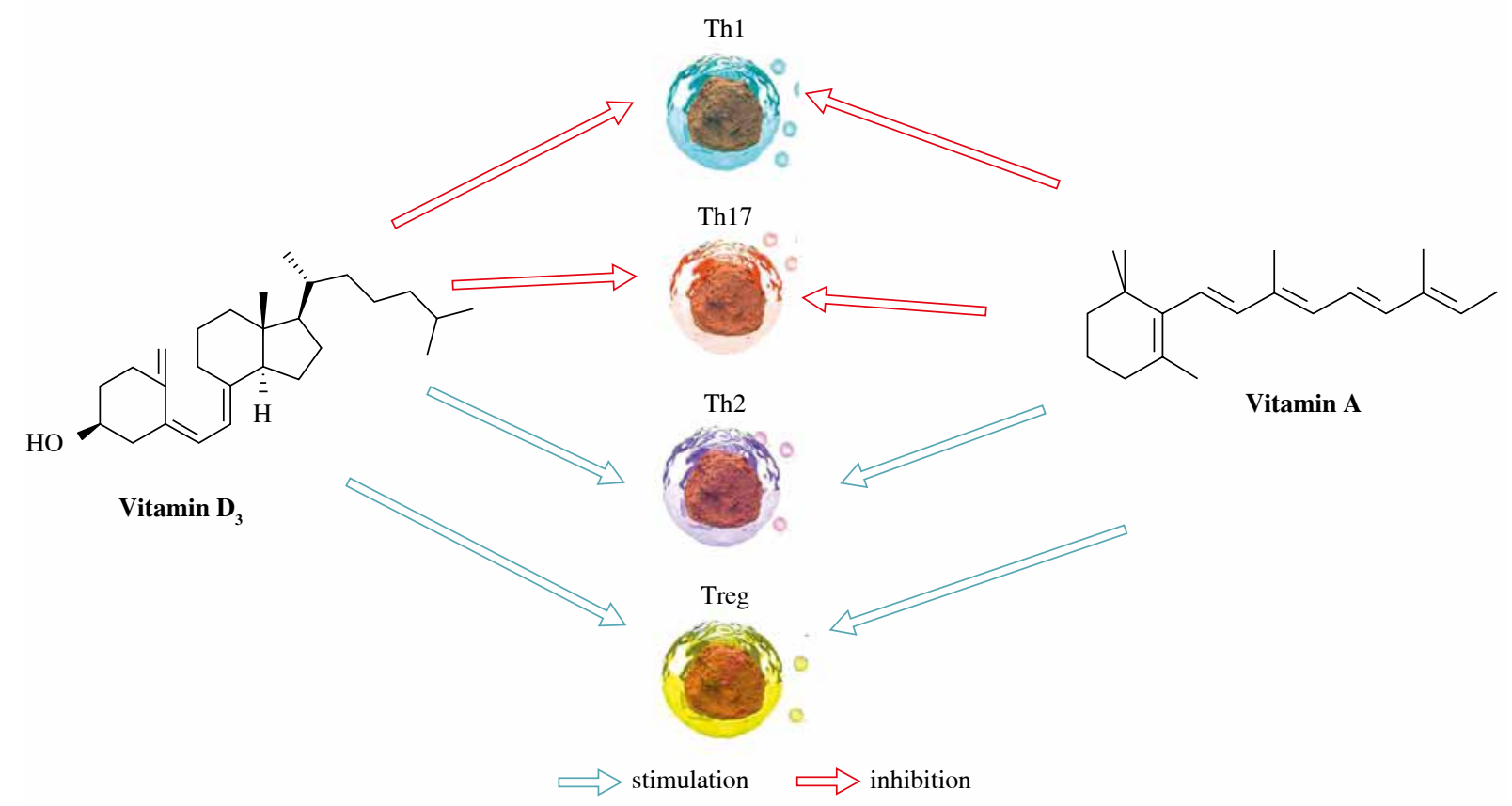

Fig. 3. Immune response polarization capacity of vitamins A and D

mers which act as a specific receptor for 9-cis-retinoic acid $[15,35]$. Since RXR proteins are known as partners for some other nuclear receptors (thyroid hormone, liver X), it is possible that due to their common RXR nuclear binding partners, the ligands vitamins $\mathrm{D}$ and $\mathrm{A}$ antagonize each other's effects when excess of vitamin A may make vitamin D supplementation less effective.

\section{Conclusions}

As the interdisciplinary approach continues to develop in research, the relationship between nutrition and immunity emerges. The influence of vitamins on the immune function of the organism has been widely studied. The foregoing review highlights that vitamins $\mathrm{A}$ and $\mathrm{D}$ are vital for immune function (a schematic presentation is shown in 
Fig. 3). By analyzing immune mechanisms, these vitamins have been shown to be potent immunomodulators, and as such it would be of great interest to continue research with a view to their application as therapeutics.

\section{Acknowledgements}

This work was supported by grants of the Ministry of Education and Science of the Republic of Serbia (projects number 175102 and 175056) and by Medical Faculty Niš (project number 30).

\section{The authors declare no conflict of interest.}

\section{References}

1. Wu D, Lewis ED, Pae M, et al. (2018): Nutritional modulation of immune function: analysis of evidence, mechanisms, and clinical relevance. Front Immunol 9: 3160.

2. Calder PC (2020): Nutrition, immunity and COVID-19. BMJ Nutr Prev Health 3: 74-92.

3. Childs CE, Calder PC, Miles EA (2019): Diet and immune function. Nutrients 11: 1933.

4. Cooper EL, Ma MJ (2017): Understanding nutrition and immunity in disease management. J Tradit Complement Med 7: 386-391.

5. Sassi F, Tamone C, D'Amelio P (2018): Vitamin D: nutrient, hormone, and immunomodulator. Nutrients 10: 1656.

6. Chirumbolo S, Bjorklund G, Sboarina A, et al. (2017): The role of vitamin $\mathrm{D}$ in the immune system as a pro-survival molecule. Clin Ther 39: 894-916.

7. Dzopalic T, Vucevic D, Tomic S, et al. (2011): 3,10-Dihydroxy-decanoic acid, isolated from royal jelly, stimulates Th1 polarising capability of human monocyte-derived dendritic cells. Food Chem 126: 1211-1217.

8. Larange A, Cheroutre H (2016): Retinoic acid and retinoic acid receptors as pleiotropic modulators of the immune system. Annu Rev Immunol 34: 369-394.

9. He L, Zhou M, Li YC (2019): Vitamin D/vitamin D receptor signaling is required for normal development and function of group 3 innate lymphoid cells in the gut. iScience 17: 119131.

10. Jones LH, Cook PC, Ivens AC, et al. (2015): Modulation of dendritic cell alternative activation and function by the vitamin A metabolite retinoic acid. Int Immunol 27: 589-596.

11. Kim EW, De Leon A, Jiang Z, et al. (2019): Vitamin A metabolism by dendritic cells triggers an antimicrobial response against mycobacterium tuberculosis. mSphere 4: e00327-19.

12. Vanherwegen AS, Eelen G, Ferreira GB, et al. (2019): Vitamin D controls the capacity of human dendritic cells to induce functional regulatory $\mathrm{T}$ cells by regulation of glucose metabolism. J Steroid Biochem Mol Biol 187: 134-145.

13. Beijer MR, Kraal G, den Haan JM (2014): Vitamin A and dendritic cell differentiation. Immunology 142: 39-45.

14. Moise AR, Noy N, Palczewski K, et al. (2007): Delivery of retinoid-based therapies to target tissues. Biochemistry 46 : 4449-4458.

15. Pino-Lagos K, Guo Y, Noelle RJ (2010): Retinoic acid: a key player in immunity. Biofactors 36: 430-436.
16. Wculek SK, Cueto FJ, Mujal AM, et al. (2020): Dendritic cells in cancer immunology and immunotherapy. Nat Rev Immunol 20: 7-24.

17. Dzopalic T, Rajkovic I, Dragicevic A, et al. (2012): The response of human dendritic cells to co-ligation of pattern-recognition receptors. Immunol Res 52: 20-33.

18. Kaiko GE, Horvat JC, Beagley KW, et al. (2008): Immunological decision-making: how does the immune system decide to mount a helper T-cell response? Immunology 123: 326-338.

19. Beijer MR, Molenaar R, Goverse G, et al. (2013): A crucial role for retinoic acid in the development of Notch-dependent murine splenic CD8- CD4- and CD4+ dendritic cells. Eur J Immunol 43: 1608-1616.

20. Sun CM, Hall JA, Blank RB, et al. (2007): Small intestine lamina propria dendritic cells promote de novo generation of Foxp3 T reg cells via retinoic acid. J Exp Med 204: 17751785.

21. Martin-Orozco N, Muranski P, Chung Y, et al. (2009): $\mathrm{T}$ helper 17 cells promote cytotoxic $\mathrm{T}$ cell activation in tumor immunity. Immunity 31: 787-798.

22. Iwata M, Eshima Y, Kagechika H (2003): Retinoic acids exert direct effects on T cells to suppress Th1 development and enhance Th2 development via retinoic acid receptors. Int Immunol 15: 1017-1025.

23. Ma Y, Chen Q, Ross AC (2005): Retinoic acid and polyriboinosinic:polyribocytidylic acid stimulate robust anti-tetanus antibody production while differentially regulating type 1/type 2 cytokines and lymphocyte populations. J Immunol 174: 7961-7969.

24. Lovett-Racke AE, Racke MK (2002): Retinoic acid promotes the development of Th2-like human myelin basic proteinreactive T cells. Cell Immunol 215: 54-60.

25. Brown CC, Esterhazy D, Sarde A, et al. (2015): Retinoic acid is essential for Th1 cell lineage stability and prevents transition to a Th17 cell program. Immunity 42: 499-511.

26. Huang Z, Liu Y, Qi G, et al. (2018): Role of vitamin A in the immune system. J Clin Med 7: 258.

27. Raverdeau M, Mills KH (2014): Modulation of T cell and innate immune responses by retinoic acid. J Immunol 192: 2953-2958.

28. Rampal R, Awasthi A, Ahuja V (2016): Retinoic acid-primed human dendritic cells inhibit Th9 cells and induce Th1/Th17 cell differentiation. J Leukoc Biol 100: 111-120.

29. Takahashi H, Kanno T, Nakayamada S, et al. (2012): TGF-beta and retinoic acid induce the microRNA miR-10a, which targets Bcl-6 and constrains the plasticity of helper T cells. Nat Immunol 13: 587-595.

30. Dzopalic T, Bozic-Nedeljkovic B, Jurisic V (2019): Function of innate lymphoid cells in the immune-related disorders. Hum Cell 32: 231-239.

31. Spits H, Di Santo JP (2011): The expanding family of innate lymphoid cells: regulators and effectors of immunity and tissue remodeling. Nat Immunol 12: 21-27.

32. Gasteiger G, Rudensky AY (2014): Interactions between innate and adaptive lymphocytes. Nat Rev Immunol 14: 631-639.

33. Goverse G, Labao-Almeida C, Ferreira M, et al. (2016): Vitamin A controls the presence of RORgamma+ innate lymphoid cells and lymphoid tissue in the small intestine. J Immunol 196: 5148-5155.

34. Ochel A, Tiegs G, Neumann K (2019): Type 2 innate lymphoid cells in liver and gut: from current knowledge to future perspectives. Int J Mol Sci 20: 1896. 
35. Spencer SP, Wilhelm C, Yang Q, et al. (2014): Adaptation of innate lymphoid cells to a micronutrient deficiency promotes type 2 barrier immunity. Science 343: 432-437.

36. Mora JR, Iwata M, von Andrian UH (2008): Vitamin effects on the immune system: vitamins $\mathrm{A}$ and $\mathrm{D}$ take centre stage. Nat Rev Immunol 8: 685-698.

37. Chen S, Yang Y, Yan X, et al. (2012): Influence of vitamin A status on the antiviral immunity of children with hand, foot and mouth disease. Clin Nutr 31: 543-548.

38. Elom MO, Okafor FC, Eyo JE (2014): Vitamin A supplementation of malaria-infected pregnant women and infant birth weight outcomes a case study of Ebonyi State, Nigeria. Gastro 2: 109.

39. Mayo-Wilson E, Imdad A, Herzer K, et al. (2011): Vitamin A supplements for preventing mortality, illness, and blindness in children aged under 5: systematic review and meta-analysis. BMJ 343: d5094.

40. Vuletic A, Konjevic G, Milanovic D, et al. (2010): Antiproliferative effect of 13-cis-retinoic acid is associated with granulocyte differentiation and decrease in cyclin B1 and Bcl-2 protein levels in G0/G1 arrested HL-60 cells. Pathol Oncol Res 16: 393-401.

41. McGee M (2020): Vitamin D: insufficiency, uncertainty and achievability. Int J Vitam Nutr Res 90: 1-4.

42. Sigmundsdottir H, Pan J, Debes GF, et al. (2007): DCs metabolize sunlight-induced vitamin D3 to 'program' T cell attraction to the epidermal chemokine CCL27. Nat Immunol 8: 285-293.

43. Shin DM, Yuk JM, Lee HM, et al. (2010): Mycobacterial lipoprotein activates autophagy via TLR2/1/CD14 and a functional vitamin D receptor signalling. Cell Microbiol 12: 16481665.

44. Zeng Y, Luo M, Pan L, et al. (2020): Vitamin D signaling maintains intestinal innate immunity and gut microbiota: potential intervention for metabolic syndrome and NAFLD. Am J Physiol Gastrointest Liver Physiol 318: G542-G553.

45. Wei R, Christakos S (2015): Mechanisms underlying the regulation of innate and adaptive immunity by vitamin $D$. Nutrients 7: 8251-8260.

46. Konya V, Czarnewski P, Forkel M, et al. (2018): Vitamin D downregulates the IL-23 receptor pathway in human mucosal group 3 innate lymphoid cells. J Allergy Clin Immunol 141: 279-292.

47. Lin YD, Arora J, Diehl K, et al. (2019): Vitamin D is required for ILC3 derived IL-22 and protection from citrobacter rodentium infection. Front Immunol 10: 1.

48. Ruiter B, Patil SU, Shreffler WG (2015): Vitamins A and D have antagonistic effects on expression of effector cytokines and gut-homing integrin in human innate lymphoid cells. Clin Exp Allergy 45: 1214-1225.

49. Kongsbak M, Levring TB, Geisler C, et al. (2013): The vitamin D receptor and T cell function. Front Immunol 4: 148.

50. Baeke F, Takiishi T, Korf H, et al. (2010): Vitamin D: modulator of the immune system. Curr Opin Pharmacol 10: 482496.

51. Daniel C, Sartory NA, Zahn N, et al. (2008): Immune modulatory treatment of trinitrobenzene sulfonic acid colitis with calcitriol is associated with a change of a $\mathrm{T}$ helper (Th) 1/ Th17 to a Th2 and regulatory T cell profile. J Pharmacol Exp Ther 324: 23-33.

52. Bscheider M, Butcher EC (2016): Vitamin D immunoregulation through dendritic cells. Immunology 148: 227-236.
53. Chen S, Sims GP, Chen XX, et al. (2007): Modulatory effects of 1,25-dihydroxyvitamin D3 on human B cell differentiation. J Immunol 179: 1634-1647.

54. Thorne A, Tomić S, Pavlović B, et al. (2015): Tumour necrosis factor- $\alpha$ promotes survival and phenotypic maturation of Poly (I:C)-treated dendritic cells, but impairs their Th1 and Th17 polarizing capability. Cytotherapy 17: 633-646.

55. Penna G, Adorini L (2000): 1 Alpha,25-dihydroxyvitamin D3 inhibits differentiation, maturation, activation, and survival of dendritic cells leading to impaired alloreactive $\mathrm{T}$ cell activation. J Immunol 164: 2405-2411.

56. Trombetta A, Paolino S, Cutolo M (2018): Vitamin D, inflammation and immunity: review of literature and considerations on recent translational and clinical research developments. Open Rheumatol J 12 (Suppl. 1, M2): 201-213. 